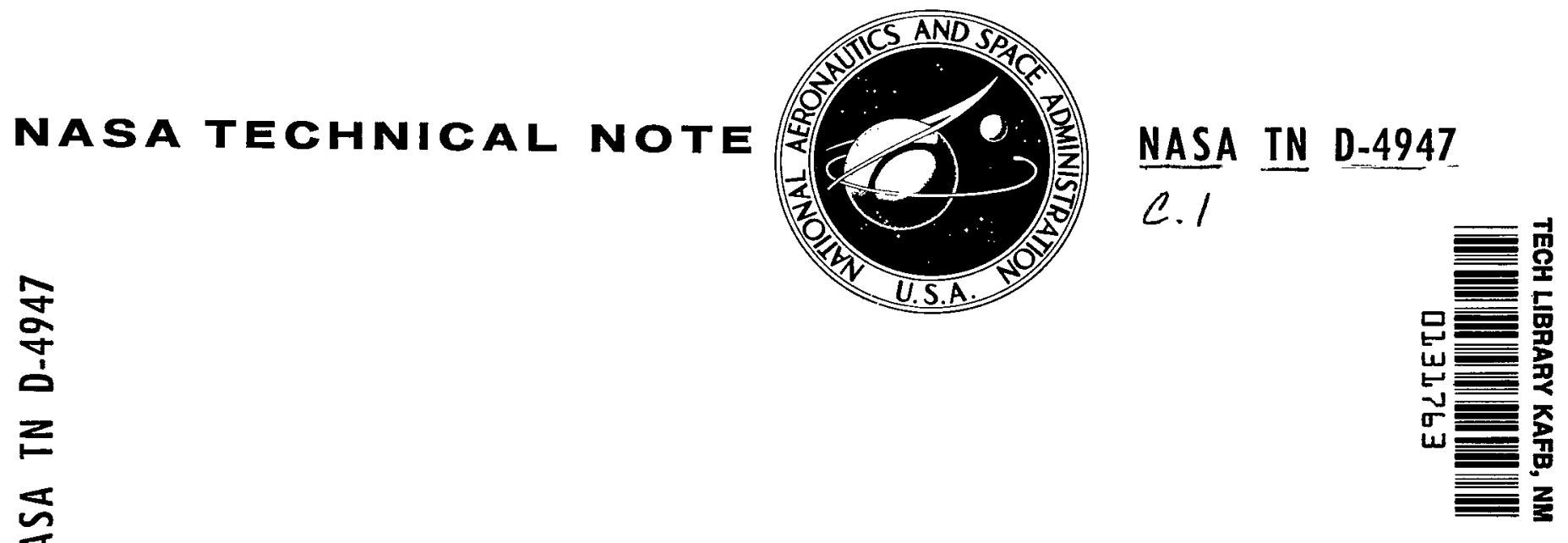

LOAN COPY: RETURN TO AFWL (WLIL-2)

KIRTLAND AFB, N MEX

NONLINEAR DECAY OF

A DISTURBANCE IN AN

UNBOUNDED VISCOUS FLUID

by Robert G. Deissler

Lewis Research Center

Cleveland, Ohio

NATIONAL AERONAUtics AND SPACE ADMINISTRATION - WASHINGTON, D. C. - DECEMBER 1968 
By Robert G. Deissler

Lewis Research Center

Cleveland, Ohio 


\section{ABSTRACT}

The nonlinear decay of a disturbance was calculated by an iterative solution to the Navier-Stokes equations. The general expressions obtained for successive approximations were suitable for machine computations, within the limitations of the computer capacity. For the initial condition, a three-dimensional cosine distribution with two harmonic terms was assumed. The nonlinear interaction of these harmonic terms then produced new harmonics. As the iteration process proceeded, a large number of harmonics, at wave numbers higher than, lower than, or the same as the original ones were generated. This process appeared to be similar to that occurring in the early stages of the development of turbulence by flow through a grid. 


\title{
NONLINEAR DECAY OF A DISTURBANCE IN AN UNBOUNDED VISCOUS FLUID
}

\author{
by Robert G. Deissler \\ Lewis Research Center
}

\section{SUMMARY}

The nonlinear decay of a disturbance was calculated by an iterative solution to the Navier-Stokes equations. The general expressions obtained for successive approximations were suitable for machine computations, within the limitations of the computer capacity. For the initial condition, a three-dimensional cosine distribution with two harmonic terms was assumed. The nonlinear interaction of these harmonic terms then produced new harmonics. As the iteration process proceeded, a large number of harmonics, at wave numbers higher than, lower than, or the same as the original ones were generated. This process appeared to be similar to that occurring in the early stages of the development of turbulence by flow through a grid.

\section{INTRODUCTION}

A fundamental problem in fluid mechanics is to predict what happens to a disturbance in a viscous fluid. Besides being of interest in itself, the problem is important as a first step in understanding the dynamics of turbulence. In particular, it should give an indication of the early stages of the development of turbulence from a regular disturbance, such as that produced by the flow of a fluid through a grid.

Taylor and Green (ref. 1), starting with an initial three-dimensional sine-cosine velocity distribution, obtained successive approximations for the velocity as a function of position and time by substituting known approximations into the Navier-Stokes equations. The results, which showed that small eddies can be generated from larger ones, were limited to small times and to the first few approximations for velocities. Jain (ref. 2) obtained similar results by using the initial conditions from reference 1 in a numerical finite-difference solution of the Navier-stokes equations.

In this analysis, an initial three-dimensional cosine velocity distribution, which is more convenient to work with than that of Taylor and Green, is used to obtain iterative solutions of the Navier-Stokes equations. Of particular importance is the fact that these 
initial conditions can be conveniently used with the compact Cartesian-tensor form of the Navier-Stokes equations. Analytical expressions are first obtained-for the first three approximations. In each approximation, the linear terms of the Navier-Stokes equations are considered as unknown, and the nonlinear terms as known from the preceding approximation. General expressions are then obtained which give the terms in an approximation of any order in terms of those in the preceding approximation. By using these expressions, a computer can be programmed to generate successively higher-order approximations from lower-order ones. It will later be seen that, in this process, new harmonics or eddy sizes are generated from existing ones. As the order of the approximation increases, the Reynolds number for which the solution is applicable increases. Accordingly, the upper Reynolds number for which the solution is applicable will be limited only by the computer capacity. Thus, the scope of the solution that can be feasibly obtained will be increased over that which could be obtained by carrying out the iterations algebraically by hand. The basic equations for the analysis will be considered in the next section.

\section{BASIC EQUATIONS AND INITIAL CONDITIONS}

The following is considered: given the initial velocity distribution in an unbounded viscous fluid, predict the motion at later times. The equations of motion for a viscous fluid with constant properties are, in dimensionless form,

$$
\begin{gathered}
\frac{\partial u_{i}}{\partial t}-\frac{\partial^{2} u_{i}}{\partial x_{k} \partial x_{k}}=-\frac{\partial p}{\partial x_{i}}-\frac{\partial\left(u_{i} u_{k}\right)}{\partial x_{k}} \\
\frac{\partial u_{k}}{\partial x_{k}}=0
\end{gathered}
$$

where

$$
\begin{gathered}
\mathrm{u}_{\mathrm{i}}=\frac{\mathrm{x}_{0}}{\nu} \mathrm{u}_{\mathrm{i}}^{*} \\
\mathrm{p}=\frac{\mathrm{x}_{0}^{2}}{\rho \nu^{2}} \mathrm{p} *
\end{gathered}
$$




$$
\begin{gathered}
t=\frac{\nu}{x_{0}^{2}} t^{*} \\
x_{i}=\frac{x_{i}^{*}}{x_{0}}
\end{gathered}
$$

The quantity $u_{i}^{*}$ is a velocity component, $x_{i}^{*}$ is a space coordinate, $x_{0}$ is a characteristic length, $\mathrm{t}^{*}$ is the time, $\rho$ is the density, and $\nu$ is the kinematic viscosity. (Note: stars on dimensional quantities are omitted on corresponding dimensionless quantities. Symbols are defined in the appendix.) The subscripts in equations (1) and (2) can take on the values 1,2 , or 3 , and a repeated subscript in a term indicates a summation. To obtain an explicit equation for the pressure, the divergence of equation (1) is applied to the continuity equation (2) to get

$$
\frac{\partial^{2} \mathrm{p}}{\partial \mathrm{x}_{\ell} \partial \mathrm{x}_{\ell}}=-\frac{\partial^{2}\left(\mathrm{u}_{\ell} \mathrm{u}_{\mathrm{k}}\right)}{\partial \mathrm{x}_{\ell} \partial \mathrm{x}_{\mathrm{k}}}
$$

In the remainder of the analysis, it will be convenient to use the set of equations (1) and (3), rather than (1) and (2).

The expression assumed for the initial disturbance is, in dimensionless form,

$$
u_{i}=a_{i}^{0} \cos \vec{q} \cdot \vec{x}+b_{i}^{0} \cos \vec{r} \cdot \vec{x}
$$

where

$$
\begin{array}{cc}
\mathrm{a}_{\mathrm{i}}^{0}=\frac{\mathrm{x}_{0}}{\nu} \mathrm{a}_{\mathrm{i}}^{0 *} & \mathrm{~b}_{\mathrm{i}}^{0}=\frac{\mathrm{x}_{0}}{\nu} \mathrm{b}_{\mathrm{i}}^{0 *} \\
\overrightarrow{\mathrm{q}}=\mathrm{x}_{0} \overrightarrow{\mathrm{q}}^{*} & \overrightarrow{\mathrm{r}}=\mathrm{x}_{0} \overrightarrow{\mathrm{r}}^{*}
\end{array}
$$

$a_{i}^{0 *}$ and $b_{i}^{0^{*}}$ are initial velocity amplitudes or Fourier coefficients of the disturbance, and $\overrightarrow{\mathrm{q}}^{*}$ and $\overrightarrow{\mathrm{r}}^{*}$ are initial wave number vectors. The quantities $\overrightarrow{\mathrm{q}} \cdot \overrightarrow{\mathrm{x}}$ and $\overrightarrow{\mathrm{r}} \cdot \overrightarrow{\mathrm{x}}$ are dot products (i.e., $\vec{q} \cdot \vec{x}=q_{1} x_{1}+q_{2} x_{2}+q_{3} x_{3}$ ). In order to satisfy the continuity condition (2), the $a^{\prime} s, b^{\prime} s, q^{\prime} s$, and $r^{\prime} s$ in equation (4) must be related by 
and

$$
\left.\begin{array}{l}
a_{i}^{0} q_{i}=0 \\
b_{i}^{0} r_{i}=0
\end{array}\right\}
$$

Equation (4) could be generalized to include any number of similar harmonic terms. It represents the simplest expression that can give nonzero pressure and inertia terms when substituted into equations (1) and (3). It can be readily verified that, if only one term of equation (4) were used, the continuity conditions (5) would require the pressure and inertia terms (terms on right side of eq. (1)) to be zero, so that nonlinear effects would be absent. Because the present study is mainly concerned with nonlinear effects, two har monic terms are included in equation (4).

\section{ANALYSIS FOR FIRST THREE APPROXIMATIONS}

As a first approximation for $\mathrm{u}_{\mathrm{i}}$ at any time

$$
u_{i}=a_{i} \cos \vec{q} \cdot \vec{x}+b_{i} \cos \vec{r} \cdot \vec{x}
$$

where $a_{i}$ and $b_{i}$ are functions of time, the forms for which will be determined from the equations of motion. The form of equation (6) has been chosen so that it will reduce to equation (4) for $\mathrm{t}=0$.

Substituting equation (6) into the right side of (3) yields

$$
\frac{\partial^{2}\left(u_{\ell} u_{k}\right)}{\partial x_{\ell} \partial x_{k}}=q_{\ell} b_{\ell} r_{k} a_{k} \cos (\vec{q}-\vec{r}) \cdot \vec{x}-q_{\ell} b_{\ell} r_{k} a_{k} \cos (\vec{q}+\vec{r}) \cdot \vec{x}
$$

For obtaining equation (7) and subsequent equations, the following identities are used:

$$
\begin{aligned}
& \sin A \sin B=\frac{1}{2}[\cos (A-B)-\cos (A+B)] \\
& \sin A \cos B=\frac{1}{2}[\sin (A-B)+\sin (A+B)]
\end{aligned}
$$




$$
\cos A \cos B=\frac{1}{2}[\cos (A-B)+\cos (A+B)]
$$

In order to be consistent with equations (3) and (7), it is assumed that

$$
p=a \cos (\vec{q}-\vec{r}) \cdot \vec{x}+b \cos (\vec{q}+\vec{r}) \cdot \vec{x}
$$

Substituting equations (7), (11), and (5) into (3) and equating coefficients of like cosine terms yield

$$
\begin{aligned}
& a=\frac{q_{\ell} l^{b} l_{k} a_{k}}{q^{2}-2 q_{\ell} l_{\ell}+r^{2}} \\
& b=-\frac{q_{\ell} l_{l} r_{k} a_{k}}{q^{2}+2 q} \ell^{r} l^{2}+r^{2}
\end{aligned}
$$

where $q$ and $r$ are the magnitudes of the initial wave number vectors $\vec{q}$ and $\vec{r}$ (i.e., $q^{2}=q_{i} q_{i}$ ). Substituting equations (6) and (8) to (13) into the right side of (1) gives

$$
\begin{aligned}
-\frac{\partial p}{\partial x_{i}}-\frac{\partial\left(u_{i} u_{k}\right)}{\partial x_{k}}= & {\left[\frac{q_{\ell} b_{\ell} r_{k} a_{k}}{q^{2}-2 q_{\ell} r_{\ell}+r^{2}}\left(q_{i}-r_{i}\right)+\frac{1}{2}\left(a_{i} q_{\ell} b_{\ell}-b_{i} r_{\ell} a_{\ell}\right)\right] \sin (\vec{q}-\vec{r}) \cdot \vec{x} } \\
& +\left[-\frac{q_{\ell} b_{l} r_{k} a_{k}}{q^{2}+2 q_{\ell} r_{\ell}+r^{2}}\left(q_{i}+r_{i}\right)+\frac{1}{2}\left(a_{i} q_{\ell} b_{\ell}+b_{i} r_{\ell} a_{\ell}\right)\right] \sin (\vec{q}+\vec{r}) \cdot \vec{x}
\end{aligned}
$$

In order to be consistent with equations (1), (4), and (14), it is assumed that, for the second approximation for $u_{i}$,

$$
u_{i}=a_{i} \cos \vec{q} \cdot \vec{x}+b_{i} \cos \vec{r} \cdot \vec{x}+c_{i} \sin (\vec{q}-\vec{r}) \cdot \vec{x}+d_{i} \sin (\vec{q}+\vec{r}) \cdot \vec{x}
$$

From the continuity condition (2) and equation (15),

$$
a_{i} q_{i}=0
$$




$$
\begin{gathered}
b_{i} r_{i}=0 \\
c_{i}\left(q_{i}-r_{i}\right)=0 \\
d_{i}\left(q_{i}+r_{i}\right)=0
\end{gathered}
$$

To solve equation (1) by iteration, it is assumed that the linear viscous term $\partial^{2} u_{i} / \partial x_{k} \partial x_{k}$, as well as $\partial u_{i} / \partial t$, is unknown at each stage of the iteration. Considering $\partial^{2} u_{i} / \partial x_{k} \partial x_{k}$ as unknown should give a more accurate answer than would be obtained by considering it as known from the preceding approximation, as has been done in previous work. It will be shown that the present scheme gives a series of negative exponentials in time, rather than a power series, as was obtained in reference 1 . Substituting equations (15) and (14) into the left and right sides of equation (1), respectively, gives, after equating coefficients of like sine and cosine terms,

$$
\frac{\partial a_{i}}{\partial t}+q^{2} a_{i}=0
$$

Using the initial condition (4) yields

$$
a_{i}=a_{i}^{0} e^{-q^{2} t}
$$

Similarly,

$$
b_{i}=b_{i}^{0} e^{-r^{2} t}
$$

The use of equations (16) and (17) in the right side of equation (1) (i.e., eq. (14)) then gives

$$
\frac{\partial c_{i}}{\partial t}+\left(q^{2}-2 q_{k} r_{k}+r^{2}\right) c_{i}
$$

$$
=\left[\frac{q_{\ell} b_{\ell}^{0} r_{k^{2}} a_{k}^{0}}{q^{2}-2 q_{\ell} r_{\ell}+r^{2}}\left(q_{i}-r_{i}\right)+\frac{1}{2}\left(a_{i}^{0} q_{\ell} b_{\ell}^{0}-b_{i}^{0} r_{\ell} a_{\ell}^{0}\right)\right] e^{-\left(q^{2}+r^{2}\right) t}
$$


or, again by use of initial condition (4),

$$
\begin{array}{r}
c_{i}=-\frac{1}{4 q_{k} r_{k}}\left[\frac{2 a_{k}^{0} r_{k} b_{l}^{0} q_{l}}{q^{2}-2 q_{\ell} r_{l}+r^{2}}\left(q_{i}-r_{i}\right)+a_{i}^{0} b_{l}^{0} q_{\ell}-b_{i}^{0} a_{\ell}^{0} r_{l}\right] \\
\times\left[e^{-\left(q^{2}+r^{2}\right) t}-e^{-\left(q^{2}-2 q_{k} r_{k}+r^{2}\right) t}\right]
\end{array}
$$

Similarly,

$$
\begin{array}{r}
d_{i}=\frac{1}{4 q_{k} r_{k}}\left[-\frac{2 a_{k}^{0} r_{k} b_{\ell}^{0} q_{\ell}}{q^{2}+2 q_{\ell} r_{\ell}+r^{2}}\left(q_{i}+r_{i}\right)+a_{i}^{0} b_{\ell}^{0} q_{\ell}+b_{i}^{0} a_{\ell}^{0} r_{\ell}\right] \\
\times\left[e^{-\left(q^{2}+r^{2}\right) t}-e^{-\left(q^{2}+2 q_{k} r_{k}+r^{2}\right) t}\right]
\end{array}
$$

The third approximation is obtained in similar fashion by substituting the second approximation into the right sides of equations (1) and (3).

The first, second, and third approximations for $u_{i}$ may be summarized as follows:

First approximation:

$$
u_{i}=a_{i}^{0} e^{-q^{2} t} \cos \vec{q} \cdot \vec{x}+b_{i}^{0} e^{-r^{2} t} \cos \vec{r} \cdot \vec{x}
$$

Second approximation: 


$$
\begin{aligned}
u_{i}=a_{i}^{0} e^{-q^{2} t} \cos \vec{q} \cdot \vec{x}+b_{i}^{0} e^{-r^{2} t} \cos \vec{r} \cdot \vec{x} & -\frac{1}{4 r_{k} q_{k}}\left[\frac{2 a_{k}^{0} r_{k} b_{\ell}^{0} q_{\ell}}{q^{2}-2 q_{\ell} r_{\ell}+r^{2}}\left(q_{i}-r_{i}\right)+a_{i}^{0} b_{\ell}^{0} q_{\ell}-b_{i}^{0} a_{\ell}^{0} r_{\ell}\right] \\
\times & {\left[e^{-\left(q^{2}+r^{2}\right) t}-e^{-\left(q^{2}-2 q_{k} r_{k}+r^{2}\right) t}\right] \sin (\vec{q}-\vec{r}) \cdot \vec{x} } \\
+ & \frac{1}{4 q_{k} r_{k}}\left[-\frac{2 a_{k}^{0} r_{k} b_{\ell}^{0} q_{\ell}}{q^{2}+2 q_{\ell} r_{\ell}+r^{2}}\left(q_{i}+r_{i}\right)+a_{i}^{0} b_{\ell}^{0} q_{\ell}+b_{i}^{0} a_{\ell}^{0} r_{\ell}\right] \\
& \times\left[e^{-\left(q^{2}+r^{2}\right) t}-e^{-\left(q^{2}+2 q_{k} r_{k}+r^{2}\right) t}\right] \sin (\vec{q}+\vec{r}) \cdot \vec{x}
\end{aligned}
$$

Third approximation:

$$
\begin{aligned}
u_{i}= & \left(a_{i}^{0} e^{-q^{2} t}+\left[\frac{1}{2 q^{2}}\left(b_{l}^{0} q_{\ell} c_{k}^{0} q_{k}+c_{\ell}^{0} q_{\ell} b_{k}^{0} q_{k}\right) q_{i}-\frac{1}{2}\left(b_{i}^{0} c_{k}^{0} q_{k}+c_{i}^{0} b_{k}^{0} q_{k}\right)\right]\right. \\
& \times\left\{-\frac{1}{2 r^{2}}\left[e^{\left(-q^{2}-2 r^{2}\right) t}-e^{-q^{2} t}\right]-\frac{1}{2 q_{k} r_{k}-2 r^{2}}\left[e^{\left(-q^{2}+2 q_{k} r_{k}-2 r^{2}\right) t}-e^{-q^{2} t}\right]\right\} \\
& +\left[\frac{1}{2 q^{2}}\left(b_{\ell}^{0} q_{\ell} d_{k}^{0} q_{k}+d_{\ell}^{0} q_{\ell} b_{k}^{0} q_{k}\right) q_{i}-\frac{1}{2}\left(b_{i}^{0} d_{k}^{0} q_{k}+d_{i}^{0} b_{k}^{0} q_{k}\right)\right]\left\{-\frac{1}{2 r^{2}}\left[e^{\left(-q^{2}-2 r^{2}\right) t}-e^{-q^{2} t}\right]\right.
\end{aligned}
$$

(Cont. on next page)

8 


$$
\begin{aligned}
& \left.\left.+\frac{1}{2 q_{k} r_{k}+2 r^{2}}\left[e^{\left(-q^{2}-2 q_{k} r_{k}-2 r^{2}\right) t}-e^{-q^{2} t}\right]\right\}\right) \cos \vec{q} \cdot \vec{x} \\
& +\left(b_{i}^{0} e^{-r^{2} t}+\left[-\frac{1}{2 r^{2}}\left(a_{\ell}^{0} l_{\ell} c_{k}^{0} r_{k}+c_{l^{0}}^{0} \ell^{a_{k}^{0} r_{k}}\right) r_{i}+\frac{1}{2}\left(a_{i}^{0} c_{k}^{0} r_{k}+c_{i}^{0} a_{k}^{0} r_{k}\right)\right]\right. \\
& \times\left\{-\frac{1}{2 q^{2}}\left[e^{\left(-2 q^{2}-r^{2}\right) t}-e^{-r^{2} t}\right]+\frac{1}{2 q^{2}-2 q_{k} r_{k}}\left[e^{\left(-2 q^{2}+2 q_{k} r_{k}-r^{2}\right) t}-e^{-r^{2} t}\right]\right\} \\
& +\left[\frac{1}{2 r^{2}}\left(a_{\ell}^{0}{ }^{0} l^{d_{k}} r_{k}+d_{\ell}^{0} l_{l}{ }^{a}{ }_{k}^{0} r_{k}\right) r_{i}+\frac{1}{2}\left(-a_{i}^{0} d_{k}^{0} r_{k}-d_{i}^{0} a_{k}^{0} r_{k}\right)\right]\left\{-\frac{1}{2 q^{2}}\left[e^{\left(-2 q^{2}-r^{2}\right) t}-e^{-r^{2} t}\right]\right. \\
& \left.\left.+\frac{1}{2 q^{2}+2 q_{k} r_{k}}\left[e^{\left(-2 q^{2}-2 q_{k} r_{k}-r^{2}\right) t}-e^{-r^{2} t}\right]\right\}\right) \cos \vec{r} \cdot \vec{x} \\
& -\frac{1}{4 q_{k} r_{k}}\left[\frac{2 b_{l}^{0} q_{l} e_{k}^{0} r_{k}}{q^{2}-2 q_{k} r_{k}+r^{2}}\left(q_{i}-r_{i}\right)+a_{i}^{0} b_{k}^{0} q_{k}-b_{i}^{0} a_{k}^{0} r_{k}\right] \\
& \times\left[e^{-\left(q^{2}+r^{2}\right) t}-e^{-\left(q^{2}-2 q_{k} r_{k}+r^{2}\right) t}\right] \sin (\vec{q}-\vec{r}) \cdot \vec{x}
\end{aligned}
$$




$$
\begin{aligned}
& +\frac{1}{4 q_{k} r_{k}}\left[-\frac{2 a_{l}^{0} e^{b_{k}^{0} q_{k}}}{q^{2}+2 q_{k} r_{k}+r^{2}}\left(q_{i}+r_{i}\right)+a_{i}^{0} b_{k}^{0} q_{k}+b_{i}^{0} a_{k}^{0} r_{k}\right] \\
& \times\left[e^{-\left(q^{2}+r^{2}\right) t}-e^{-\left(q^{2}+2 q_{k} r_{k}+r^{2}\right) t}\right] \sin (\vec{q}+\vec{r}) \cdot \vec{x} \\
& +\left[-\frac{a_{\ell}^{0} r_{\ell} c_{k}^{0} q_{k}}{4 q^{2}-4 q_{k} r_{k}+r^{2}}\left(2 q_{i}-r_{i}\right)-\frac{1}{2}\left(a_{i}^{0} c_{k}^{0} q_{k}-c_{i}^{0} a_{k}^{0} r_{k}\right)\right] \\
& \times\left\{\frac{1}{2 q^{2}-4 q_{k} r_{k}}\left[e^{\left(-2 q^{2}-r^{2}\right) t}-e^{-\left(4 q^{2}-4 q_{k} r_{k}+r^{2}\right) t}\right]\right. \\
& \left.-\frac{1}{2 q^{2}-2 q_{k} r_{k}}\left[e^{\left(-2 q^{2}+2 q_{k} r_{k}-r^{2}\right) t}-e^{-\left(4 q^{2}-4 q_{k} r_{k}+r^{2}\right) t}\right]\right\} \cos (2 \vec{q}-\vec{r}) \cdot \vec{x} \\
& +\left[\frac{a_{\ell}^{0} r_{l} d_{k}^{0} q_{k}}{4 q^{2}+4 q_{k} r_{k}+r^{2}}\left(2 q_{i}+r_{i}\right)-\frac{1}{2}\left(a_{i}^{0} d_{k}^{0} q_{k}-d_{i}^{0} a_{k}^{0} r_{k}\right)\right] \\
& \times\left\{\frac{1}{2 q^{2}+4 q_{k} r_{k}}\left[e^{\left(-2 q^{2}-r^{2}\right) t}-e^{-\left(4 q^{2}+4 q_{k} r+r^{2}\right) t}\right]\right.
\end{aligned}
$$

(Cont. on next page)

10 


$$
\begin{aligned}
& \left.-\frac{1}{2 q^{2}+2 q_{k} r_{k}}\left[e^{\left(-2 q^{2}-2 q_{k} r_{k}-r^{2}\right) t}-e^{-\left(4 q^{2}+4 q_{k} r_{k}+r^{2}\right) t}\right]\right\} \cos (2 \vec{q}+\vec{r}) \cdot \vec{x} \\
& +\left[-\frac{c_{\ell}^{0} l_{l} b_{k}^{0} q_{k}}{q^{2}-4 q_{k} r_{k}+4 r^{2}}\left(q_{i}-2 r_{i}\right)-\frac{1}{2}\left(c_{i}^{0} b_{k}^{0} q_{k}-b_{i}^{0} c_{k}^{0} r_{k}\right)\right] \\
& \times\left\{\frac{1}{-4 q_{k} r_{k}+2 r^{2}}\left[e^{\left(-q^{2}-2 r^{2}\right) t}-e^{-\left(q^{2}-4 q_{k} r_{k}+4 r^{2}\right) t}\right]\right. \\
& \left.+\frac{1}{2 q_{k} r_{k}-2 r^{2}}\left[e^{\left(-q^{2}+2 q_{k} r_{k}-2 r^{2}\right) t}-e^{-\left(q^{2}-4 q_{k} r_{k}+4 r^{2}\right) t}\right]\right\} \cos (\vec{q}-2 \vec{r}) \cdot \vec{x} \\
& +\left[\frac{d_{\ell}^{0} r_{l} b_{k}^{0} q_{k}}{q^{2}+4 q_{k} r_{k}+4 r^{2}}\left(q_{i}+2 r_{i}\right)-\frac{1}{2}\left(d_{i}^{0} b_{k}^{0} q_{k}+b_{i}^{0} d_{k}^{0} r_{k}\right)\right] \\
& \times\left\{\frac{1}{4 q_{k} r_{k}+2 r^{2}}\left[e^{\left(-q^{2}-2 r^{2}\right) t}-e^{-\left(q^{2}+4 q_{k} r_{k}+4 r^{2}\right) t}\right]\right. \\
& \left.-\frac{1}{2 q_{k} r_{k}+2 r^{2}}\left[e^{\left(-q^{2}-2 q_{k} r_{k}-2 r^{2}\right) t}-e^{-\left(q^{2}+4 q_{k} r_{k}+4 r^{2}\right) t}\right]\right\} \cos (\vec{q}+2 \vec{r}) \cdot \vec{x}
\end{aligned}
$$




$$
\begin{aligned}
& +\left[-\frac{2 r_{i}}{r^{2}} c_{l^{0}}^{0} l^{d_{k}^{0} r_{k}}+\left(c_{i}^{0} d_{k}^{0}+d_{i}^{0} c_{k}^{0}\right) r_{k}\right]\left[\frac{e^{\left(-2 q^{2}-2 r^{2}\right) t}-e^{-4 r^{2} t}}{-q^{2}+r^{2}}\right. \\
& \left.-\frac{e^{\left(-2 q^{2}-2 q_{k} r_{k}-2 r^{2}\right) t}-e^{-4 r^{2} t}}{-2 q^{2}-2 q_{k} r_{k}+2 r^{2}}-\frac{e^{\left(-2 q^{2}+2 q_{k} r_{k}-2 r^{2}\right) t}-e^{-4 r^{2} t}}{-2 q^{2}+2 q_{k} r_{k}+2 r^{2}}\right] \sin 2 \vec{r} \cdot \vec{x} \\
& +\left[\frac{2 q_{i}}{q^{2}} c_{\ell}^{0} q_{\ell} d_{k}^{0} q_{k}-\left(c_{i}^{0} d_{k}^{0}+d_{i}^{0} c_{k}^{0}\right) q_{k}\right]\left[\frac{e^{\left(-2 q^{2}-2 r^{2}\right) t}-e^{-4 q^{2} t}}{q^{2}-r^{2}}\right. \\
& \left.-\frac{e^{\left(-2 q^{2}-2 q_{k} r_{k}-2 r^{2}\right) t}-e^{-4 q^{2} t}}{2 q^{2}-2 q_{k} r_{k}-2 r^{2}}-\frac{e^{\left(-2 q^{2}+2 q_{k} r_{k}-2 r^{2}\right) t}-e^{-4 q^{2} t}}{2 q^{2}+2 q_{k} r_{k}-2 r^{2}}\right] \sin 2 \vec{q} \cdot \vec{x}
\end{aligned}
$$

where $a_{i}^{0}$ and $b_{i}^{0}$ are given by the initial condition (4), and

$$
\begin{aligned}
& c_{i}^{0}=-\frac{1}{4 q_{k} r_{k}}\left[\frac{2 a_{k}^{0} r_{k} b_{\ell}^{0} q_{l}}{q^{2}-2 q_{\ell} r_{\ell}+r^{2}}\left(q_{i}-r_{i}\right)+a_{i}^{0} b_{\ell}^{0} q_{\ell}-b_{i}^{0} a_{\ell}^{0} r_{\ell}\right] \\
& d_{i}^{0}=\frac{1}{4 a_{k} r_{k}}\left[-\frac{2 a_{k}^{0} r_{k} b_{\ell}^{0} q_{\ell}}{q^{2}+2 q_{\ell} r_{\ell}+r^{2}}\left(q_{i}+r_{i}\right)+a_{i}^{0} b_{\ell}^{0} q_{\ell}+b_{i}^{0} a_{\ell}^{0} r_{\ell}\right]
\end{aligned}
$$

It is evident that the number and complexity of terms in the expression for $u_{i}$ increase rapidly as the order of the approximation increases. This happens because each term in a particular approximation is multiplied by every other one when it is substituted into the nonlinear terms of equations (1) and (3); that is, each sine or cosine term interacts with every other one to produce more terms. If, on the other hand, the nonlinear terms in equations (1) and (3) were neglected (low Reynolds number), the first approximation (eq. (21)) would be an exact solution of (1) and (3). 


\section{GENERAL EXPRESSIONS FOR NONLINEAR PRODUCTION OF HARMONICS}

Because of the proliferation of new harmonic terms as the order of the approximation increases, it appears impracticable to generate approximations much higher than the third analytically. Rather, it would seem better to obtain general expressions for the new terms produced by the interaction of pairs of harmonic terms in a particular approximation. It should then be possible to program a computer to generate successive approximations from preceding ones. These general expressions for the nonlinear interaction of pairs of harmonic terms will be considered next.

Examination of the terms in the first three approximations for $u_{i}$ (eqs. (21) to (23)) shows that they are of the general form

$$
u_{i}=\sum_{\vec{\kappa}}\left(A_{i, \vec{\kappa}}^{c} \cos \vec{\kappa} \cdot \vec{x}+A_{i, \vec{\kappa}}^{s} \sin \vec{\kappa} \cdot \vec{x}\right)
$$

where

$$
A_{i, \vec{\kappa}}^{c}=\sum_{q} a_{i, \vec{\kappa}, q^{c}} e^{-b_{\vec{\kappa}}^{c}, q^{t}}
$$

and

$$
\mathrm{A}_{\mathrm{i}, \vec{\kappa}}^{\mathrm{s}}=\sum_{\mathrm{r}} \mathrm{a}_{\mathrm{i}, \vec{\kappa}, \mathrm{r}^{\mathrm{s}}} \mathrm{e}_{\vec{\kappa}, \mathrm{r}^{\mathrm{t}}}^{\mathrm{s}}
$$

The superscripts $s$ and $c$ appear in coefficients of sine and cosine terms, respectively. The indices $\vec{\kappa}, \mathrm{q}$, and $\mathrm{r}$ take on as many values as are required to represent the terms of the particular approximation.

When equation (26) is substituted into the nonlinear terms of equations (1) and (3), each term of equation (26) is multiplied by (or interacts with) every other term. Thus, sine - sine, sine - cosine, and cosine - cosine products must be considered. Consider, first, the terms generated by the interaction of two terms

and

$$
\left.\begin{array}{l}
A_{i, \vec{n}}^{c} \cos \vec{n} \cdot \vec{x} \\
A_{i, \vec{m}}^{c} \cos \vec{m} \cdot \vec{x}
\end{array}\right\}
$$


Thus, the sum

$$
A_{i, \vec{n}}^{c} \cos \vec{n} \cdot \vec{x}+A_{i, \vec{m}}^{c} \cos \vec{m} \cdot \vec{x}
$$

where

$$
\begin{aligned}
& A_{i, \vec{n}}^{c}=\sum_{q} a_{i, \vec{n}, q^{c}}^{c} e^{-b_{\vec{n}, q}^{c} t} \\
& A_{i, \vec{m}}^{c}=\sum_{r} a_{i, \vec{m}, r^{c}} e^{-b_{\vec{m}, r^{t}}^{c}}
\end{aligned}
$$

is substituted for $u_{i}$ in the right sides of equations (3) and (1). The manipulations are similar to those used for obtaining the first and second approximations in the last section. Because it is necessary to obtain the additive contribution to $u_{i}$, which results from the interaction of the two harmonic terms in equation (30), $u_{i}$ is replaced on the left side of equation (1) by $u_{i, \vec{n}}^{c}, \vec{m}$, which represents that contribution. For the initial condition on $u_{i, \vec{n}}^{c}, \vec{m}, u_{i, \vec{n}, \vec{m}}^{c, c}$ is set equal to zero when $t=0$. (Initial condition (4) will be satisfied later when the totality of terms contributing to $u_{i}$ is considered.) The expression for $u_{i, \vec{n}, \vec{m}}^{c, c}$ is, then,

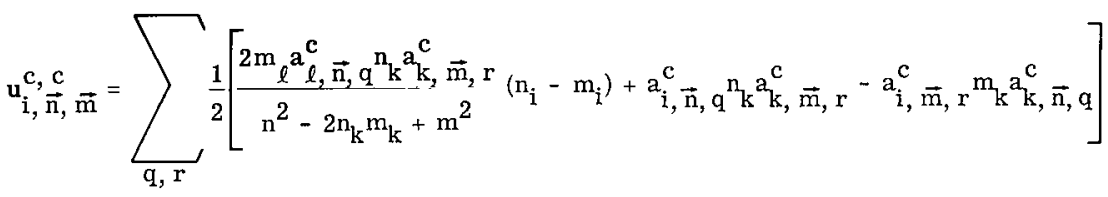

$$
\begin{aligned}
& \times \frac{e^{-\left(b_{\vec{m}, r}^{c}+b_{n}^{c}, q\right) t}-e^{-\left(n^{2}-2 n_{k} m_{k}+m^{2}\right) t}}{s i n(\vec{n}-\vec{m}) \cdot \vec{x}}
\end{aligned}
$$

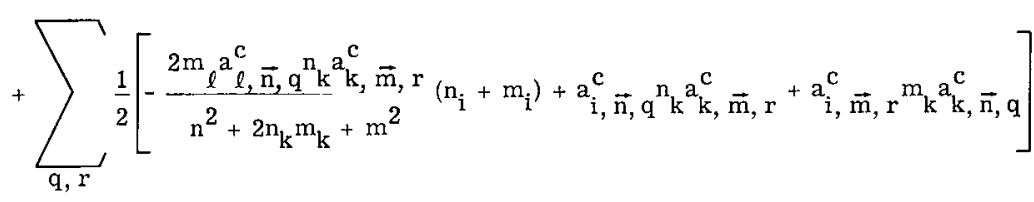

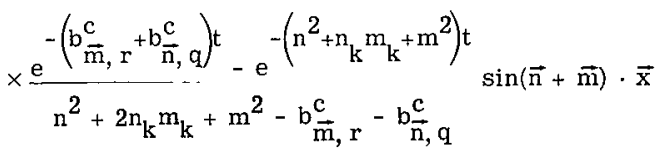


Similarly, the interaction of terms like

and

$$
\left.\begin{array}{l}
A_{i, \vec{n}}^{s} \sin \vec{n} \cdot \vec{x} \\
A_{i, \vec{m}}^{s} \sin \vec{m} \cdot \vec{x}
\end{array}\right\}
$$

where

and

$$
A_{i, \vec{n}}^{s}=\sum_{q} a_{i, \bar{n}, q^{s}} e^{-b_{\vec{n}, q}^{s}}
$$

results in

$$
A_{i, \vec{m}}^{s}=\sum_{r} a_{i, \vec{m}, r^{s}} e^{-b_{\vec{m}}^{s}, r^{t}}
$$

$$
\begin{aligned}
& u_{i, \vec{n}, \vec{m}}^{s, s}=\sum_{q, r} \frac{1}{2}\left[\frac{2 m_{l} a_{l, \vec{n}, q}^{s} n_{k} a_{k, \vec{m}, r}^{s}}{n^{2}-2 n_{k} m_{k}+m^{2}}\left(n_{i}-m_{i}\right)+a_{i, \vec{n}, q}^{s} n_{k} a_{k}^{s}, \vec{m}, r-a_{i, \vec{m}}^{s}, m_{k} a_{k, \vec{n}, q}^{s}\right]
\end{aligned}
$$

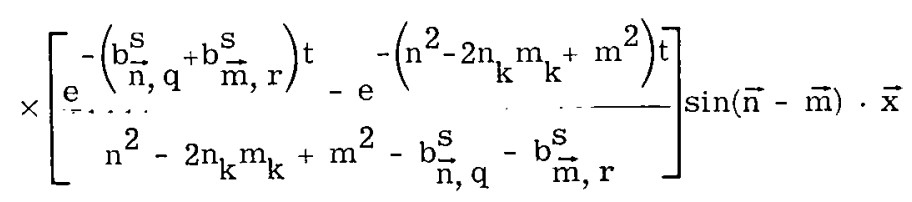

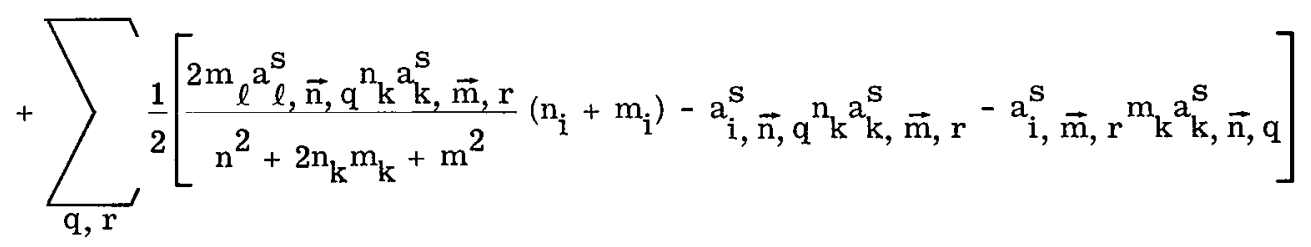

$$
\begin{aligned}
& \times\left[\begin{array}{c}
e^{-\left(b_{\vec{n}, q}^{s}+b_{\vec{m}, r}^{s}\right) t}-e^{-\left(n^{2}+2 n_{k} m_{k}+m^{2}\right) t} \\
n^{2}+2 n_{k} m_{k}+m^{2}-b_{\vec{n}, q}^{s}-b_{\vec{m}, r}^{s}
\end{array}\right] \sin (\vec{n}+\vec{m}) \cdot \vec{x}
\end{aligned}
$$


Finally, the interaction of terms like

and

$$
\left.\begin{array}{l}
A_{i, \vec{n}}^{s} \sin \vec{n} \cdot \vec{x} \\
A_{i, \vec{m}}^{c} \cos \vec{m} \cdot \vec{x}
\end{array}\right\}
$$

where $A_{i, \vec{n}}^{S}$ and $A_{i, \vec{m}}^{c}$ are defined by equations (34) and (30), results in

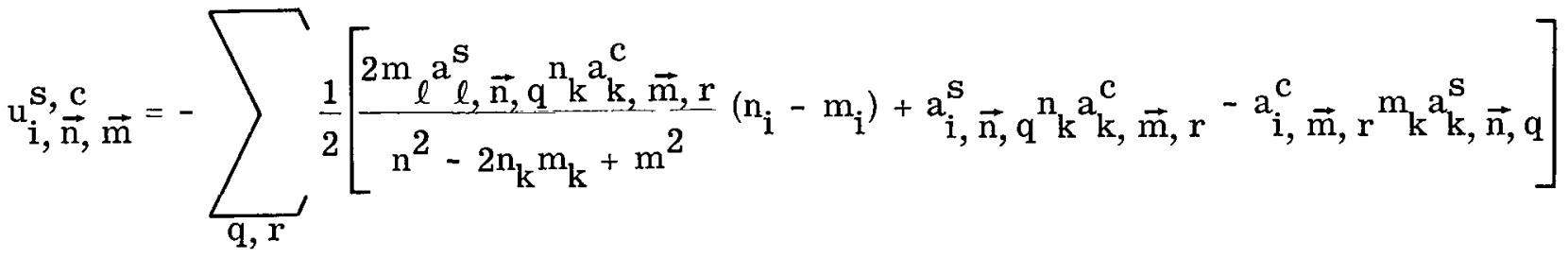

$$
\begin{aligned}
& \times\left[\frac{e^{-\left(b_{\vec{n}, q^{s}}^{\left.+b_{\vec{m}, r}^{c}\right) t}\right.}-e^{-\left(n^{2}-2 n_{k} m_{k}+m^{2}\right) t}}{n^{2}-2 n_{k} m_{k}+m^{2}-b_{\vec{n}, q}^{s}-b_{\vec{m}, r}^{c}}\right] \cos (\vec{n}-\vec{m}) \cdot \vec{x}
\end{aligned}
$$

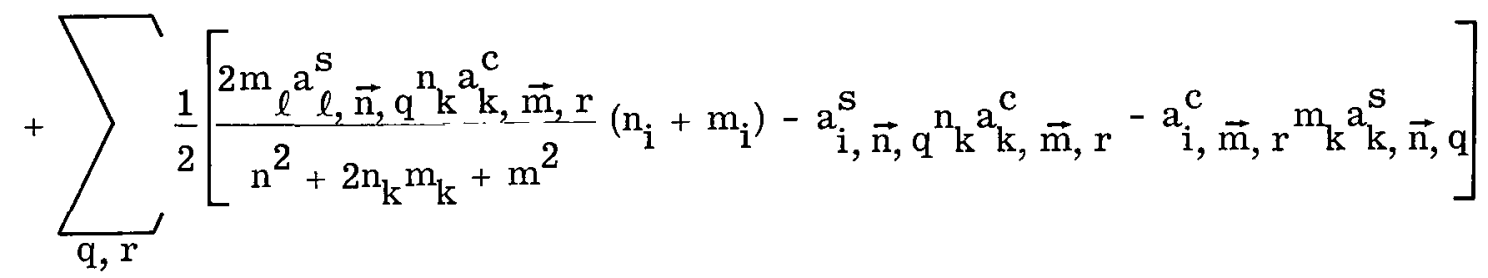

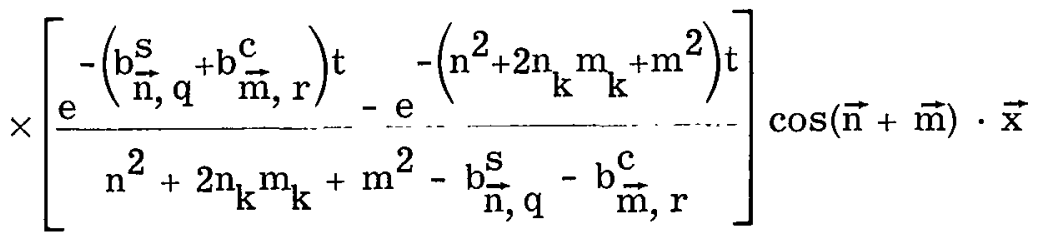

The successive approximations to the velocity components $u_{i}$ may now be calculated. If the $\mathrm{v}^{\text {th }}$ approximation is known, equations (33), (37), and (39) give contributions to the $v+1^{\text {st }}$ approximation, which are due to the presence (or interaction) of pairs of harmonic terms in the $v^{\text {th }}$ approximation. Thus, if the $v^{\text {th }}$ approximation for $u_{i}$ is given by 


$$
\begin{aligned}
& u_{i}^{(v)}=\ldots+A_{i, \vec{n}}^{c} \cos \vec{n} \cdot \vec{x}+A_{i, \vec{n}}^{s} \sin \vec{n} \cdot \vec{x} \\
& +\ldots+\ldots+A_{i, \vec{m}}^{c} \cos \vec{m} \cdot \vec{x}+A_{i, \vec{m}}^{s} \sin \vec{m} \cdot \vec{x}+\ldots
\end{aligned}
$$

then the $v+1^{\text {st }}$ approximation is

$$
\begin{aligned}
& u_{i}^{(v+1)=\ldots+u_{i, \vec{n}, \vec{m}}^{c, c}}+\ldots+u_{i, \vec{n}, \vec{m}}^{s, s}+\ldots+u_{i, \vec{n}, \vec{m}}^{s, c}+\ldots \\
& \quad+\quad \begin{array}{l}
u_{i, \vec{m}, \vec{n}}^{s, c}+\ldots+a_{i}^{0} e^{-q^{2} t} \cos \vec{q} \cdot \vec{x}+b_{i}^{0} e^{-r^{2} t} \cos \vec{r} \cdot \vec{x}
\end{array}
\end{aligned}
$$

where the $u_{i}^{(}, \vec{n}, \vec{m}^{\prime}$ s are given by the equations (33), (37), and (39).

The last two terms in equation (41) are the first approximation (eq. (21)) and are included in equation (41) because, whereas every other term is regenerated at each stage in the iteration process, the terms of the first approximation are not so regenerated. That is the case because of the assumed initial condition $u_{i,}^{c}, \vec{n}, \vec{m}=0$ when $t=0$. The same result could be obtained by letting $u_{i, \vec{n}, \vec{m}}^{c, c}=a_{i}^{0} \cos \vec{q} \cdot \vec{x}+b_{i}^{0} \cos \vec{r} \cdot \vec{x}$ at $t=0$ when the two interacting terms include the first approximation. However, it is simpler to use equation (33) throughout as it stands and to include the first approximation in equation (41).

In obtaining equation (41) from (40), the presence (or interaction) of each pair of harmonic terms in equation (40) produces a term $u_{i, \vec{m}}^{()} \vec{n}$ in (41). Thus, each term in equation (40) interacts once with every other term. Because of the continuity condition (2) (see also eq. (5)) no contributions are obtained by the interaction of a term with itself. Thus, if there are $s$ terms in the $\mathrm{v}^{\text {th }}$ approximation, the $\mathrm{v}+1^{\text {st }}$ approximation will have $(s-1)+(s-2)+\ldots .+1=(s / 2)(s-1)$ contributions, like $u_{i}() \vec{n}, \vec{m}$, plus the first approximation.

With the velocity components $u_{i}$ known to some degree of approximation, the space-averaged values of $u_{i}^{2}$ can be calculated. Because $u_{i}$ is given by equation (26), squaring, integrating over a cycle, and using the orthogonality property of sines and cosines result in 


$$
\begin{aligned}
\overline{u_{i}^{2}} & =\frac{1}{2 \pi} \int_{-\pi}^{\pi}\left(\sum_{\vec{\kappa}} A_{i, \vec{\kappa}}^{\mathrm{c}} \cos \vec{\kappa} \cdot \overrightarrow{\mathrm{x}}+\mathrm{A}_{i, \vec{\kappa}}^{\mathrm{s}} \sin \vec{\kappa} \cdot \overrightarrow{\mathrm{x}}\right)^{2} \mathrm{~d}(\vec{\kappa} \cdot \overrightarrow{\mathrm{x}}) \\
& =\sum_{\vec{\kappa}} \frac{1}{2}\left[\left(\mathrm{~A}_{\mathrm{i}, \vec{\kappa}}^{\mathrm{c}}\right)^{2}+\left(\mathrm{A}_{\dot{\mathrm{i}}, \vec{\kappa}}^{\mathrm{s}}\right)^{2}\right]
\end{aligned}
$$

\section{RESULTS AND DISCUSSION}

If the velocity distribution in a fluid is given by a sum of harmonic terms as in equation (26), then the presence (or nonlinear interaction) of each pair of terms produces new harmonics given by equations (29) to (39). Each pair of terms then present interacts to produce still more harmonic terms or eddy sizes. It is evident that there will result a very large number of eddy sizes, or a situation resembling turbulent flow. It should be pointed out that, because of wave number vectors $\vec{n}-\vec{m}$ and $\vec{n}+\vec{m}$, which occur, for instance, in equation (33), the generated harmonics can have either higher or lower wave numbers than the original ones. Moreover, when some of the generated harmonics interact with the original ones, new harmonics can be generated with the same wave numbers as the original ones.

One might ask how a random turbulence could develop from a regular disturbance produced, for example, by flow through a grid with equal spacings. It has been pointed out earlier that because of continuity, harmonics of the same wave number and/or intensity vectors will not interact nonlinearly. However, if there are small random disturbances in the flow upstream of the grid or small imperfections in the grid, the eddies produced by the grid will not be identical; therefore, they can interact to produce new harmonics. Because of the random nature of the small upstream distrubances, or grid imperfections, the resulting flow will be random or turbulent.

Figure 1 shows calculated kinetic energy per unit mass for a disturbance plotted as a function of dimensionless time. First, second, third, and fourth approximations are shown. The initial condition for the disturbance is given by equation (4), where the values of $a_{i}^{0}, b_{i}^{0}, q_{i}$, and $r_{i}$ are shown in the figure. The values of the initial vectors were chosen to satisfy continuity and to give nonzero interaction terms $\left\langle a_{i}^{0} q_{i}=b_{i}^{0} r_{i}=0\right.$, $a_{i}^{0} r_{i} \neq 0$, and $\left.b_{i}^{0} q_{i} \neq 0\right)$. Disturbances having three different initial intensities are compared. The first approximation (eq. (21)) gives the linear decay that occurs when the nonlinear or inertia terms in the Navier-Stokes equation are neglected. Comparison of 


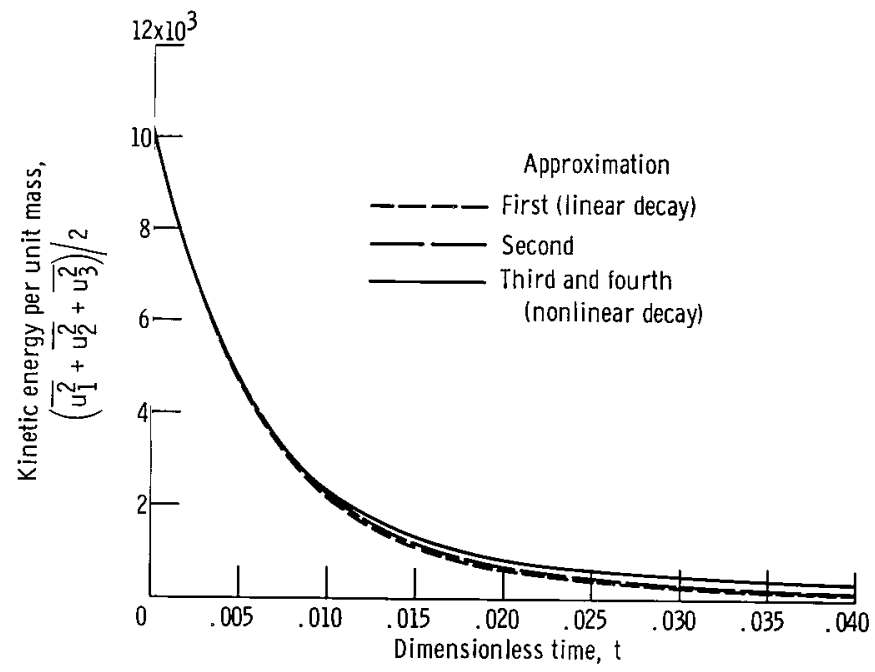

(a) Weaker disturbance Initial dimensionless Fourier coefficients: $a_{i}^{0}=20(1,-1,-2,) ; b_{i}^{0}=80(1,-2,-1)$.

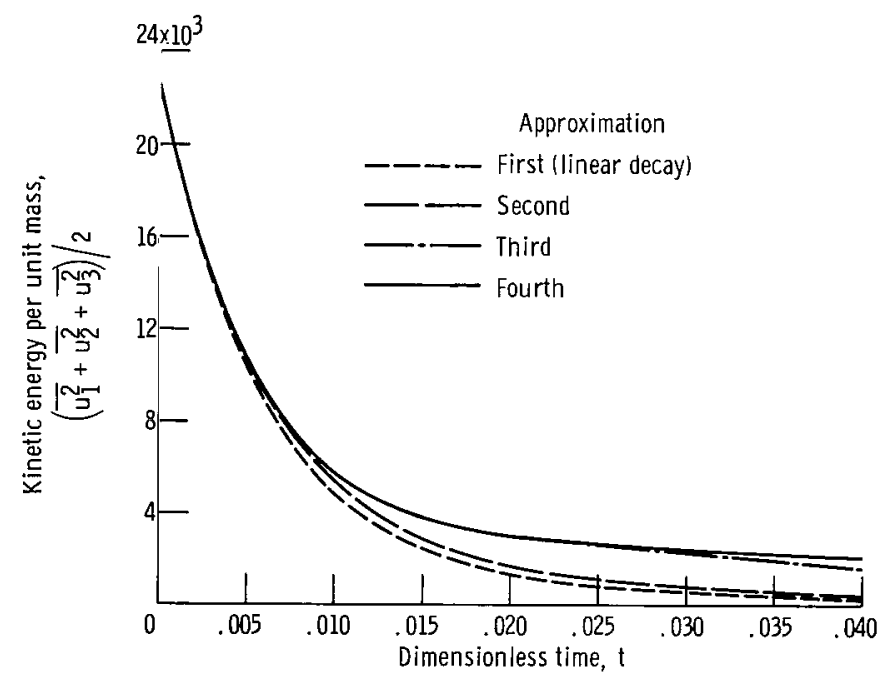

(b) Moderate disturbance. Initial dimensionless Fourier coefficients: $a_{i}^{0}=30(1,-1,-2) ; b_{1}^{0}=120(1,-2,-1)$.
$45 \times 10^{3}$

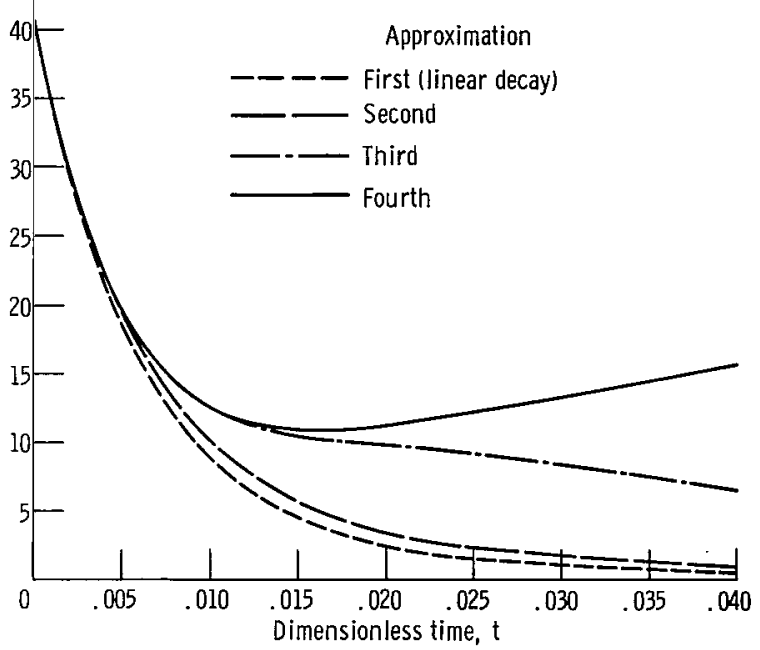

(c) Stronger disturbance. Initial dimensionless Fourier coefficients: $a_{i}^{0}=40(1,-1,-2) ; b_{i}^{0}=160(1,-2,-1)$. 
the nonlinear with the linear solutions shows that the nonlinear effects cause the disturbances to decay more slowly. It is noted that a similar result is obtained for random turbulent flow. Strongly nonlinear turbulence decays approximately as $\mathrm{t}^{-1}$, whereas the decay law for linear turbulence is closer to $t^{-5 / 2}$.

Comparison of the various approximations indicates that at large times, higher order approximations are required for the iteration process to converge (as indicated by the agreement of the third and fourth approximations) than are required at smaller times. Larger values of the initial intensity parameters indicate convergence only at small times for the first four approximations. On the other hand, smaller values of the initial intensity show convergence even at very large times. The poorer convergence of a given approximation at larger intensities is, of course, due to the stronger inertia effects that occur at high Reynolds numbers; therefore, higher approximations are required to take them into account.

To show how the energy is distributed among the various directional components, the ratios $\overline{u_{2}^{2}} / \overline{u_{1}^{2}}$ and $\overline{u_{3}^{2}} / \overline{u_{1}^{2}}$ are plotted against dimensionless time in figure 2 . The case shown corresponds to that in figure 1(b). Comparison of the curves in figure 1(b) with those in figure 2 shows that the third and fourth approximations in the curves in figure 2 converge for a smaller range of dimensionless times than do those of figure 1. Evidently, a given order approximation at a time $t$ is less accurate for the individual components than it is for the total energy. Comparison of the first (linear approximation for $\overline{u_{2}^{2}} / \overline{u_{1}^{2}}$ and for $\overline{u_{3}^{2}} / \overline{u_{1}^{2}}$ shows that the ratios can either increase or decrease with time because the different components decay viscously at different rates, depending on the initial conditions.

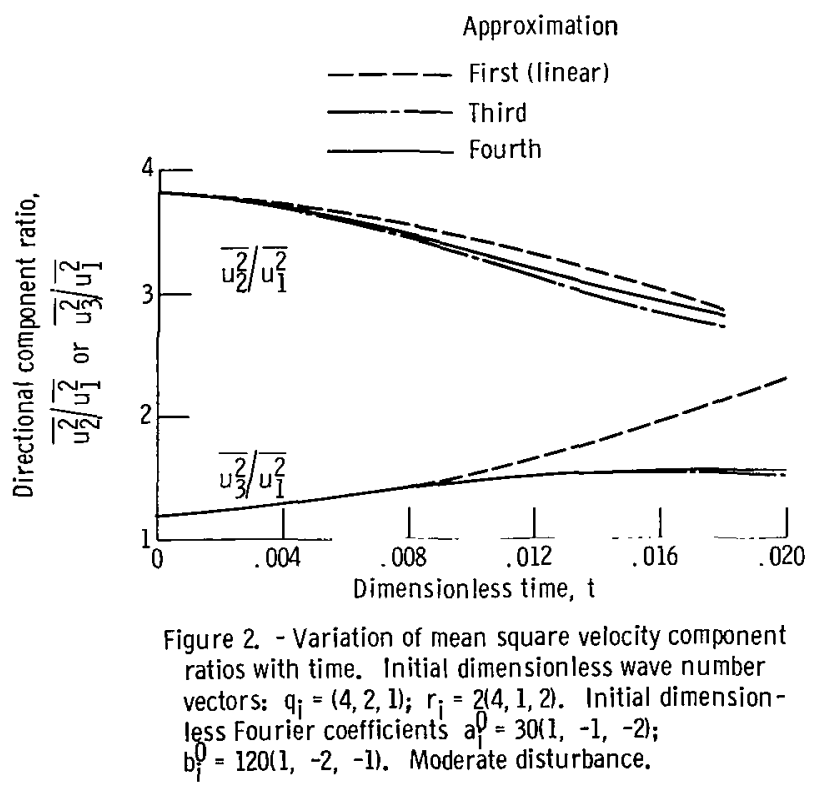


The fourth (nonlinear) approximations show the same general trends as the first approximations, but the nonlinear effects tend to bring the ratios closer to one (this is also true for $\overline{u_{3}^{2}} / \overline{u_{2}^{2}}$; that is, the nonlinear effects tend to make the disturbance more isotropic. To show how various harmonics contribute to the kinetic energy of the disturbance, kinetic energy spectra were calculated from equation (42) and plotted against dimensionless wave number $\kappa$ in figure 3. The cases shown here are the same as those in figure 1. Results are given for several dimensionless times for which the iteration process has converged (see fig. 1). For $t=0$, there are, of course, only two spikes or harmonics in the spectrum because of the assumed initial condition (eq. (4)). For the later times shown, particularly for the stronger disturbances, a large number of other har-

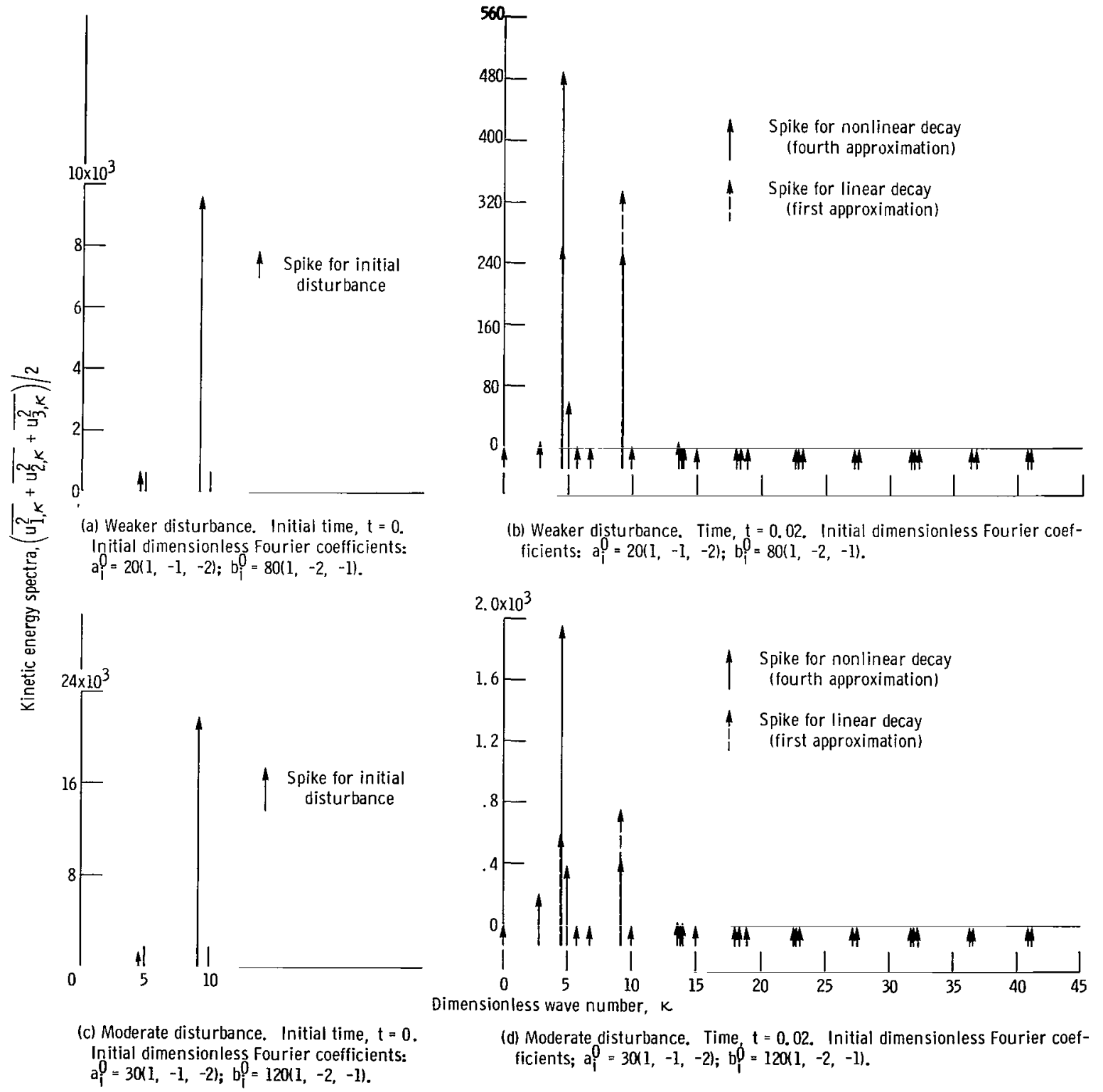

Figure 3. - Spectrum of mean kinetic energy per unit mass, Components of initial wave number vectors: $q_{i}=(4,2,1) ; r_{i}=2(4,1,2)$. 


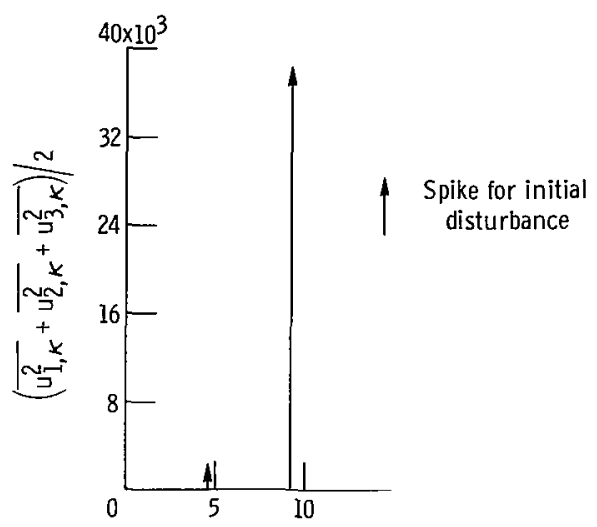

(e) Stronger disturbance. Initial time, $t=0$. Initial dimensionless Fourier coefficients: $a_{1}=40(1,-1,-2) ; b_{i}^{0}=160(1,-2,-1)$.

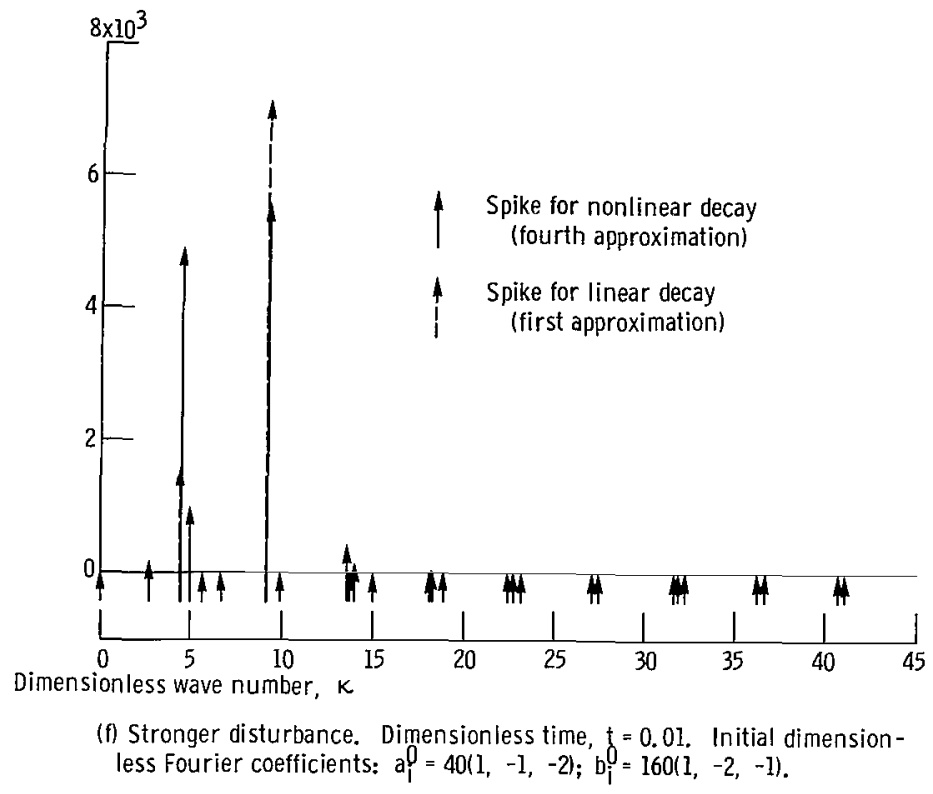

Figure 3. - Concluded.

monics have developed because of the interaction between contributions at various wave numbers. Although some of the generated harmonics are strong, many of them are too weak to have an appreciable effect on the disturbance, so that their representations lie close to the horizontal axis. By increasing the strength of the disturbance, more of the harmonics will have appreciable strengths. However, the fourth approximation will then not be sufficient to obtain convergence of the iterative process. Unfortunately, the computer used for the calculations did not have enough storage capacity to carry out the approximations higher than the fourth.

For comparison with the nonlinear results just discussed, the spikes for the linear solution are shown dashed in figures $3(\mathrm{~b}),(\mathrm{d})$, and (f). In this case, no new harmonics are generated. The original harmonics simply decay independently. Comparison of the solid and dashed lines shows that the harmonics at the original wave numbers are greatly affected by nonlinear interactions; that is, many of the eddies, generated or destroyed by nonlinear effects, have the same wave numbers as those in the original disturbance. It might be mentioned that, in order for that to occur, it is necessary to go to at least the third approximation because the second approximation produces only contributions that are at higher or lower wave numbers than the original ones (eq. (22)).

Although the harmonics generated by nonlinear effects can occur at either high or low wave numbers, most of the significant generated harmonics in the calculated results, particularly those for the weaker disturbances, tend to lie in the low wave number re- 
gion. The difficulty of generating significant harmonics at the higher wave numbers appears to be related to the fact that viscous effects in the high wave number region tend to prevent their formation. As the disturbance strength increases, however, the tendency to form significant harmonics in the higher wave number region appears to increase.

\section{CONCLUDING REMARKS}

An initial three-dimensional disturbance consisting of two harmonics can decay nonlinearly and generate new harmonics at wave numbers greater than, smaller than, or the same as, those of the original harmonics. If either the wave number vectors or the amplitude vectors of the two initial harmonics have the same direction, however, the continuity condition requires that there be no interaction. In that case or if nonlinear effects are ignored by neglecting the inertia terms in the Navier-Stokes equation, the original harmonics will simply decay independently.

In general, nonlinear effects cause the disturbance to decay more slowly. At low Reynolds numbers there is, of course, no significant difference between the linear and nonlinear solutions.

The ratios of the directional components of the mean square velocities changed with time. This was caused by differences in the rates of viscous (linear) decay of the various directional components and by transfer of energy between the components by nonlinear action. Whereas the viscous effects could change the component ratios in either direction (depending on the initial conditions) the nonlinear effects always tended to bring the ratios closer to one or to make the disturbance more isotropic.

For a given approximation, the iterative solution for the nonlinear case was most accurate at small times and/or small Reynolds numbers of the disturbance. As either time or the Reynolds number increased, the order of the approximation that was required to obtain convergence of the iterative process increased.

The generation of a range of harmonics in the early stages of the development of turbulence by flow through a grid appears to be similar to the production of har monics in the present analysis. Random turbulence can evidently develop by flow through a grid because of small random disturbances in the flow upstream of the grid or small imperfections in the grid. Random harmonics are then generated as a result of the interaction of the slightly different intensities and wave numbers of the disturbances in the flow from the grid.

\section{Lewis Research Center,}

National Aeronautics and Space Administration, Cleveland, Ohio, September 9, 1968, 129-01-05-20-22. 


\section{APPENDIX - SYMBOLS}
$\mathrm{A}_{\mathrm{i}, \vec{\kappa}}^{\mathrm{c}}, \mathrm{A}_{\mathrm{i}, \vec{\kappa}}^{\mathrm{S}}, \mathrm{A}_{\mathrm{i}, \overrightarrow{\mathrm{n}}}^{\mathrm{c}}$,$$
A_{i, \vec{m}}^{\mathrm{c}}, A_{i, \vec{n}}^{\mathrm{s}}, A_{i, \vec{m}}^{\mathrm{s}}
$$$$
\mathrm{A}_{i, \vec{\kappa}}^{\mathrm{c}^{*}}, \mathrm{~A}_{\mathrm{i}, \vec{\kappa}}^{\mathbf{s}^{*}}
$$
$\mathrm{a}, \mathrm{b}$
$a_{i}, b_{i}, c_{i}, d_{i}$
$a_{i, \vec{\kappa}}^{c}, q^{,} a_{i, \vec{\kappa}}^{s}, r, a_{i, \vec{n}}^{c}, q^{\prime}$ $a_{i, \vec{m}}^{c}, r, a_{i, \vec{n}}^{s}, q, a_{i, \vec{m}}^{s}, r$
$a_{i}^{0}, b_{i}^{0}$
$a_{i}^{0 *}, b_{i}^{0 *}$
$a_{i}^{*}, b_{i}^{*}$
$a^{*}, b^{*}$
$\mathrm{b}_{\vec{\kappa}, \mathrm{q}}^{\mathrm{c}}, \mathrm{b}_{\vec{\kappa}}^{\mathrm{s}}, \mathrm{r}, \mathrm{b}_{\overrightarrow{\mathrm{n}}, \mathrm{q}^{\prime}}^{\mathrm{c}}$,
$\mathrm{b}_{\overrightarrow{\mathrm{m}}, \mathrm{r}}^{\mathrm{c}}, \mathrm{b}_{\overrightarrow{\mathrm{n}}, \mathrm{q}}^{\mathrm{s}}, \mathrm{b}_{\overrightarrow{\mathrm{m}}, \mathrm{r}}^{\mathrm{s}}$
$\mathrm{m}, \mathbf{n}$
$\mathrm{m}_{\mathbf{i}}, \mathrm{n}_{\mathbf{i}}$
$\mathrm{m}_{\mathrm{i}}^{*}, \mathrm{n}_{\mathrm{i}}^{*}$
$\overrightarrow{\mathrm{m}}, \overrightarrow{\mathrm{n}}$
p
$p^{*}$
$q_{i}, r_{i}$

dimensionless Fourier coefficients for velocity components, $\mathrm{x}_{0} \mathrm{~A}_{\mathrm{i}, \vec{\kappa}}^{\mathrm{c}^{*}} / \nu$

Fourier coefficients for velocity component

dimensionless Fourier coefficients for pressure fluctuation, $\mathrm{x}_{0}^{2} \mathrm{a}^{*} / \rho \nu^{2}, \mathrm{x}_{0}^{2} \mathrm{~b}^{*} / \rho \nu^{2}$ (see eq. (11))

dimensionless Fourier coefficients for velocity component, $\mathrm{x}_{0} \mathrm{a}_{\mathrm{i}}^{*} / \nu, \mathrm{x}_{0} \mathrm{~b}_{\mathrm{i}}^{*} / \nu$, etc. (see eqs. (6) and (15))

constants given by eqs. (27), (28), (31), (32), (35), and (36)

initial dimensionless Fourier coefficients for velocity components, $x_{0} a_{i}^{0 *} / \nu$ and $x_{0} b_{i}^{0 *} / \nu$ (see eq. (4))

initial Fourier coefficients for velocity components

Fourier coefficients for velocity component

Fourier coefficients for pressure fluctuation

constants given by eqs. (27), (28), (31), (32), (35), and (36)

magnitudes of dimensionless wave number vectors

components of dimensionless wave number vectors, $\mathrm{x}_{0} \mathrm{~m}_{\mathrm{i}}^{*}$,

$$
\mathrm{x}_{0} \mathrm{n}_{\mathrm{i}}^{*}
$$

components of wave number vectors

dimensionless wave number vectors

dimensionless pressure, $\mathrm{x}_{0}^{2} \mathrm{p}^{*} / \rho \nu^{2}$

pressure

components of initial dimensionless wave number vectors, $\mathrm{x}_{0} \mathrm{q}_{\mathrm{i}}^{*}, \mathrm{x}_{0} \mathrm{r}_{\mathrm{i}}^{*}$ 
$q_{i}^{*}, r_{i}^{*}$

$\vec{q}, \overrightarrow{\mathbf{r}}$

t

$t^{*}$

$\mathrm{u}_{\mathrm{i}}$

$u_{i}^{*}$

$u_{i, \vec{n}, \vec{m}}^{c, c}, u_{i, \vec{n}, \vec{m}}^{s, s}, u_{i, \vec{n}, \vec{m}}^{s, c}$

$x_{i}$

$x_{i}^{*}$

$\vec{x}$

$\mathrm{x}_{0}$

$\vec{\kappa}$

$\vec{\kappa}^{*}$

$\nu$

$\rho$

Superscripts:

c

s

* components of initial wave number vectors

initial dimensionless wave number vectors

dimensionless time, $\nu t^{*} / \mathrm{x}_{0}^{2}$

time

component of dimensionless velocity, $x_{0} u_{i}^{*} / \nu$

component of velocity

interaction terms given by eqs. (33), (37), and (39)

dimensionless position coordinate, $x_{i}^{*} / x_{0}$

position coordinate

dimensionless position vector

characteristic length

dimensionless wave number vector, $\mathrm{x}_{0} \vec{\kappa}^{*}$

wave number vector

kinematic viscosity

density

cosine terms

sine term

dimensional quantities

averaged quantities 


\section{REFERENCES}

1. Taylor, G. I.; and Green, A. E.: Mechanism of the Production of Small Eddies

from Large Ones. Proc. Roy. Soc. (London), ser. A, vol. 158, no. 895, Feb. 3, 1937, pp. 499-521.

2. Jain, P. C.: Numerical Study of the Navier-Stokes Equations for the Production of Small Eddies from Large Ones. Univ. Wisconsin, Rep. MRC-TSR-491, Univ. Wisconsin, July 1964. 


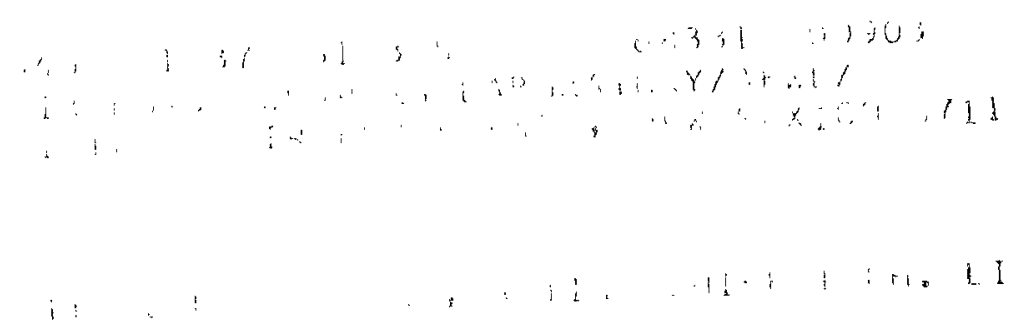

"The aeronautical and space activities of the United States shall be conducted so as to contribute. . . to the expansion of buman knowledge of phenomena in the atmosphere and space. The Administration shall provide for the widest practicable and appropriate dissemination of information concerning its activities and the results thereof."

\section{NASA SCIENTIFIC AND TECHNICAL PUBLICATIONS}

TECHNICAL REPORTS: Scientific and technical information considered important, complete, and a lasting contribution to existing knowledge.

TECHNICAL NOTES: Information less broad in scope but nevertheless of importance as a contribution to existing knowledge.

TECHNICAL MEMORANDUMS:

Information receiving limited distribution because of preliminary data, security classification, or other reasons.

CONTRACTOR REPORTS: Scientific and technical information generated under a NASA contract or grant and considered an important contribution to existing knowledge.
TECHNICAL TRANSLATIONS: Information published in a foreign language considered to merit NASA distribution in English.

SPECIAL PUBLICATIONS: Information derived from or of value to NASA activities. Publications include conference proceedings, monographs, data compilations, handbooks, sourcebooks, and special bibliographies.

\section{TECHNOLOGY UTILIZATION} PUBLICATIONS: Information on technology used by NASA that may be of particular interest in commercial and other non-aerospace applications. Publications include Tech Briefs, Technology Utilization Reports and Notes, and Technology Surveys.

Details on the availability of these publications may be obtained from:

SCIENTIFIC AND TECHNICAL INFORMATION DIVISION

NATIONAL AERONAUTICS AND SPACE ADMINISTRATION

Washington, D.C. 20546 